\title{
An uncommon case of diabetic mastopathy
}

\section{Abstract}

Introduction: Diabetic mastopathy (DMP) is an uncommon fibrous disease of the breast, most commonly found in premenopausal women with type 1 diabetes exposed to long-standing insulin therapy. Often mimicking breast cancer clinically and on imaging, this condition poses a diagnostic challenge requiring pathological confirmation.

Clinical case: A 68-year-old woman with type $1 \mathrm{DM}$ and a family history of breast cancer in paternal aunt had an abnormal screening mammogram showing a lobular nodular density in the subareolar region deep to the nipple measuring at least $2.5 \mathrm{~cm}$ in the left breast. S Ultra Sound (US) showed a hypoechoic, heterogenous mass $2 \mathrm{~cm}$ from the nipple, measuring $41 \times 13 \times 46 \mathrm{~mm}$ with relatively circumscribed margins, demonstrating some internal blood flow on Doppler. Lymph nodes were normal. Patient underwent US-guided biopsy. Pathology showed hyalinized stromal fibrosis and chronic mastitis. Within a short period of time, patient presented with a painless palpable area is the right breast. Mammography and US of right breast showed a very dense tissue in the palpable area of concern, corresponding to heterogeneous, slightly hypoechoic mass with relatively circumscribed margins measuring $38 \times 18 \times 65 \mathrm{~mm}$ with internal vascularity. It had a very similar appearance to the area that was biopsied on the left breast. Given the bilaterality and history of diabetes, a preliminary diagnosis of bilateral DMP was made. A decision was made to follow up annually with regular mammography. Later, patient developed retraction in the skin and nipple areolar complex in both breasts. Mammogram and US of both breasts revealed large densities that were relatively stable, however the skin changes prompted repeat biopsies. Thickened tissue on both breasts was excised and sent for pathology revealing features consistent with DMP.
Volume 7 Issue 2 - 2019

\author{
Rujuta Katkar,' Manasi Shah, ${ }^{2}$ Antoine \\ Makdissi' \\ 'Diabetes, Endocrinology and Metabolism, University at Buffalo, \\ USA \\ Internal Medicine, University at Buffalo, USA
}

\begin{abstract}
Correspondence: Rujuta Katkar, Diabetes, Endocrinology and Metabolism, University at Buffalo, New York, USA, 4I 23 Coventry Green Circle, Buffalo, NY-I422I, USA, Tel 20343599 76, Email katkarrujuta@yahoo.co.in
\end{abstract}

Received: February 25, 2019 | Published: April 30,2019

Discussion: Patients with DMP clinically present with painless, irregular hard unilateral or bilateral breast masses. It is rare as it represents represent $0.6 \%$ to $13 \%$ of benign lesions observed in woman with type 1 diabetes. The pathogenesis is not fully understood but many theories involving the effects of sustained hyperglycemia and glycosylated end products on the connective tissues of the breast have been proposed. Malignant transformation has not been described.

Conclusion: This case underlines the importance of considering diabetic mastopathy in the differential diagnoses when evaluating breast lesions in women with diabetes. Recognizing the presentation of this rare condition can help avoiding unnecessary surgical intervention.

Keywords: diabetes, mastopathy, breast cancer

\section{Introduction}

Diabetic mastopathy (DMP) was first described by Soler et al. ${ }^{1}$ in 1984, with a cohort of 12 women with longstanding type I diabetes mellitus [DM] who all presented with similar benign dense breast lumps. Diabetic mastopathy is an uncommon fibrous disease of the breast, most commonly found in premenopausal women with type 1 diabetes exposed to long standing insulin therapy but cases of patients with type 2 diabetes, including male patients, have also been noted. ${ }^{2}$ Here we present a case of diabetic mastopathy that was initially unilateral with contrasting findings on imaging and pathology. Often mimicking breast cancer clinically and on imaging, this condition poses a diagnostic challenge requiring pathological confirmation.

\section{Clinical case}

A 68year old woman was found to have an abnormal finding on routine mammography Patient has past history of type 1DM, peripheral neuropathy, hypothyroidism, HTN and a surgical biopsy left breast greater than 20years ago; with a family history of breast cancer in paternal aunt. Screening mammogram showed an apparent increased large lobular nodular density in the subareolar region deep to the nipple measuring atleast $2.5 \mathrm{~cm}$ in the left breast. Subsequent Ultra Sound [US] of left breast showed a mass in the region of the scar site, $2 \mathrm{~cm}$ from the nipple, measuring $41 \times 13 \times 46 \mathrm{~mm}$ demonstrating some internal blood flow on Doppler. The mass was hypoechoic and slightly heterogenous with relatively circumscribed margins. Left axilla showed normal Lymph nodes [LN]. Patient underwent US-guided biopsy. Pathology showed hyalinized stromal fibrosis and chronic mastitis. This benign pathology was discordant with mammographic and sonographic imaging findings. Therefore, surgical excision was recommended with a breast surgeon follow up. Soon thereafter, patient presented with a painless palpable area of concern in the superior and subareolar Right breast of two weeks duration. Mammography and US of right breasts showed a very dense tissue in the palpable area of concern, corresponding to heterogeneous, slightly hypoechoic mass with relatively circumscribed margins measuring $38 \times 18 \times 65 \mathrm{~mm}$ with internal vascularity. It had a very similar appearance to the area that was biopsied on the left breast in a similar position which demonstrated stromal fibrosis and chronic mastitis. A coarsely calcified vessel was noted running through the area of concern. No abnormal lymph nodes were detected. Given the bilaterality and history of diabetes, a preliminary diagnosis of bilateral diabetic mastopathy was made. A decision was made to follow up annually with regular mammography. In September 2017, patient developed retraction in the skin and nipple areolar complex in both breasts. Mammogram and US of both breasts revealed large densities which were relatively stable, however the skin changes prompted repeat biopsies. Thickened tissue on both breasts was excised and sent for pathology. Pathology showed collagenous stroma with keloidal features and an increased concentration of stromal spindle cells. The breast lobules revealed atrophy, infiltrated by mature small lymphocytes. A perivascular lymphocytic infiltrate was noted. The morphological features were consistent with diabetic mastopathy. The rest of the breast tissue revealed apocrine metaplasia and ductal dilatation. No atypia or malignancy was identified. 


\section{Discussion}

Diabetic mastopathy, also known as lymphocytic mastitis or lymphocytic mastopathy is seen occasionally in premenopausal women who have longstanding type 1 diabetes mellitus. This case underlines the importance of considering diabetic mastopathy on the list of differential diagnoses when evaluating breast lesions in a diabetic woman. Patients with DMP clinically present with painless, irregular hard unilateral or bilateral breast masses. It is rare as it represents represent $0.6 \%$ to $13 \%$ of benign lesions observed in woman with type 1 diabetes. $^{2}$ Core biopsy is recommended for diagnostic confirmation. Pathology shows dense keloid-like fibrosis and periductal, lobular, or perivascular lymphocytic infiltration. ${ }^{3-5}$ The pathogenesis is not fully understood but many theories involving the effects of sustained hyperglycemia and glycosylated end products on the connective tissues of the breast have been proposed. Also it may represent an autoimmune reaction as the histologic features are similar to those seen in other autoimmune diseases. ${ }^{6}$ There are no large series with long-term follow up, but most reports recommend annual clinical and radiological monitoring and, in case of a new lesion, an additional biopsy must be considered. Once the diagnosis is established, excision is not necessary and malignant transformation has not been described. Conservative management is adequate for the majority of patients. Lesions have a tendency to recur after resection., ${ }^{2,7}$ Recognizing the presentation of this rare condition can help avoiding unnecessary surgical intervention (Figures $1 \& 2$ ).

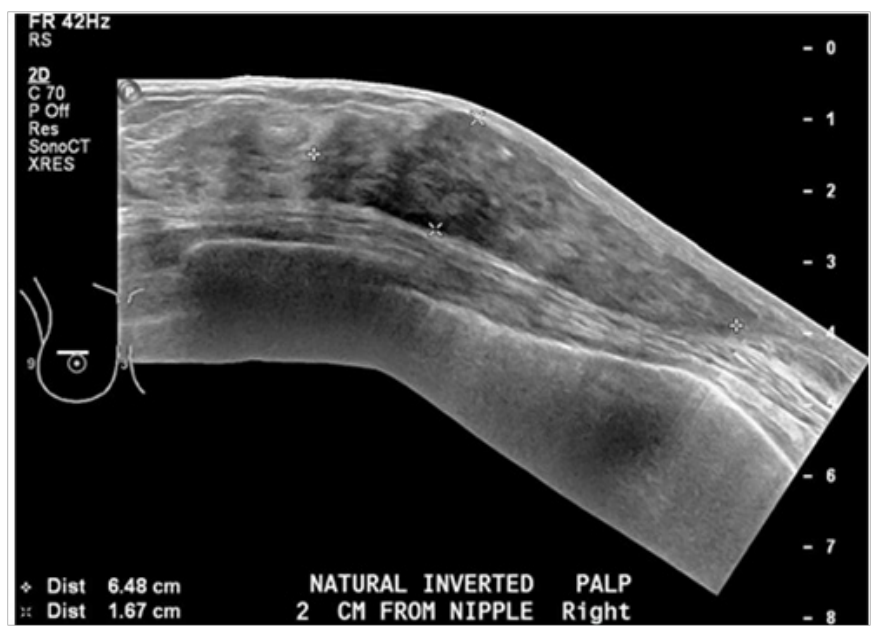

Figure I Ultrasound of the breast showing Mastopathy changes, 3 I I $\times 222 \mathrm{~mm}$ (72×72 DPI).

\section{Funding details}

N/A.

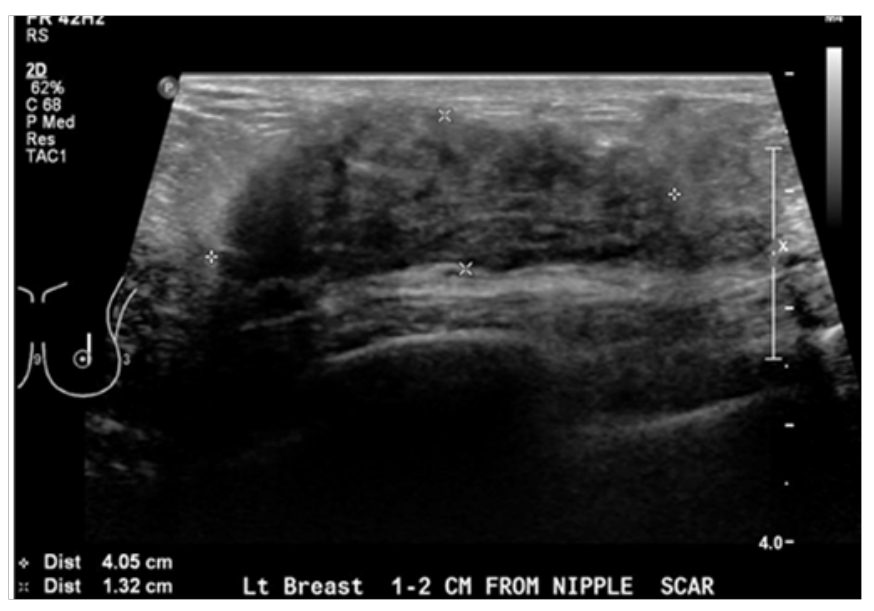

Figure 2 Ultrasound of the breast showing mastopathy changes, $321 \times 218 \mathrm{~mm}$ (72×72DPI).

\section{Acknowledgments}

None.

\section{Conflicts of interests}

Authors declare that there is no conflict of interest.

\section{References}

1. Soler NG, Khardori R. Fibrous disease of the breast, thyroiditis, and cheiroarthropathy in type I diabetes mellitus. Lancet. 1984;1(8370):193-195.

2. Leroux-Stewart J, Rabasa-Lhoret R. Diabetic Mastopathy: Case Report and Summary of Literature. Canadian Journal of Diabetes. 2014;38(5):305-306.

3. Lai EC, Chan WC, Ma TK, et al. The role of conservative treatment in idiopathic granulomatous mastitis. Breast J. 2005;11(6):454-456.

4. Gombos EC, Esserman LE, Weisberg S, et al. Granulomatous mastitis. $J$ Women's Imaging. 2004;6:136.

5. Kudva YC, Reynolds C, O'Brien T, et al. "Diabetic mastopathy," or sclerosing lymphocytic lobulitis, is strongly associated with type 1 diabetes. Diabetes Care. 2002;25(1):121-126.

6. Lammie GA, Bobrow LG, Staunton MD, et al. Sclerosing lymphocytic lobulitis of the breast-evidence for an autoimmune pathogenesis. Histopathology. 1991;19(1):13-20.

7. Sankaye S, Kachewar S. Diabetic mastopathy. Australas Med J. 2012;5(6):296-299. 